\title{
3 Research Square \\ Diversity and structure of the rhizosphere microbial communities of wild and cultivated ginseng
}

\author{
Xiaoxue Fang \\ Northeast Normal University \\ Huaying Wang \\ Northeast Normal University \\ Ling Zhao \\ Northeast Normal University \\ Manqi Wang \\ Northeast Normal University \\ Mingzhou Sun ( $\square$ sunmz100@nenu.edu.cn ) \\ Northeast Normal University
}

\section{Research Article}

Keywords: cultivated ginseng, microbial community composition, microbial diversity, phytopathogens, wild ginseng

Posted Date: June 25th, 2021

DOl: https://doi.org/10.21203/rs.3.rs-646533/v1

License: (c) (i) This work is licensed under a Creative Commons Attribution 4.0 International License. Read Full License

Version of Record: A version of this preprint was published at BMC Microbiology on January 3rd, 2022. See the published version at https://doi.org/10.1186/s12866-021-02421-w. 


\section{Abstract \\ Background}

Different plant species, even different plant varieties, will promote different combinations of microbial communities related to them. Here, the objective was to explore the differences in the rhizosphere microbial communities in the wild ginseng, farmland cultivated ginseng and understory wild ginseng. The rhizosphere soil was obtained from three type of ginsengs, namely wild ginseng (WDG), farmland cultivated ginseng (CDG) and understory wild ginseng (LXG) (all ginsengs grown in the field). The $16 \mathrm{~S}$ rRNA gene and internal transcribed spacer (ITS) region were analyzed to investigate the diversity and structure of the microbial community.

\section{Result}

We found the fungal communities were more influenced bacterial communities. There were differences in the microbial community composition under three types of ginsengs. Moreover, higher bacterial diversity and lower fungal diversity in CDG compared with WDG. Changes in rhizosphere microbial community composition and diversity of WDG and CDG may be caused by domestication. Furthermore, the relative abundance of potential phytopathogens, Chloroflexi, Fusarium and Alternaria were higher in CDG compared to WDG and LXG. This may be related to the fact that cultivated ginseng has a short life cycle and is susceptible to disease.

\section{Conclusion}

We found differences in the rhizosphere microbial community of the three types of ginsengs, and the abundance of pathogenic microorganisms is significantly different. This result provided insights into the underlying mechanisms of ginseng planting and disease resistance.

\section{Background}

The rhizosphere microbiome interacts with plants $[1,2]$. Plants rely on their rhizosphere microbes to support functions related to plant growth, development and health [3]. The association with specific of rhizosphere microorganisms is either promoted or prevented by plant roots through exudate and volatile compounds. Comparing the rhizosphere of twelve Populus trichocarpa genotypes suggested specific salicylic acid derivatives secreted by roots regulated rhizosphere microbial colonization and assembly [4]. In addition, root types (primary and secondary) and regions (root cap, lateral meristem) can also resulted in uneven distribution of microorganisms in the rhizosphere, moreover microorganisms move through the soil as the roots grow [5]. Root exudates and root morphology influence the composition and diversity of rhizosphere microbial communities, and this effect largely depends on plant species and genotype [5-7]. Root morphology and root exudates composition are distinct in different plants, therefore they affect the 
structure of the rhizosphere microbial community, which leads to the specificity of rhizosphere microbes in different plant lineages [6-8]. In addition, effect of land use changes soil microbial community [9]. Agricultural practices such as fertilizer addition also alter microbial community composition [10].

The root of ginseng (Panax ginseng C. A. Meyer.) is used as a traditional Chinese medicine to treat many diseases, due to its anti-inflammatory and antitumor compounds [11]. Ginseng belongs to the family Araliaceae that is distributed in Asia, particularly in Korea and China [12]. Wild ginseng germplasm resources are scarce due to excessive land exploitation and disruption of the environment; thus, wild ginseng has been gradually replaced by cultivated ginseng in the market [13]. Wild ginseng is considered the ancestor of cultivated ginseng, but the morphology of their roots, disease resistance and bioactive substance are significantly different $[14,15]$. There are two kinds of cultivated ginseng, farmland cultivated ginseng and understory wild ginseng. Farmland cultivated ginseng is planted in farmland that was once forested with human management, such as spraying pesticides and there the ginseng grows very quickly. In contrast, understory wild ginseng refers to farmland cultivated ginseng seeds or seedlings grown under natural forest conditions for many years with little human interference; its morphology and intrinsic quality of roots are similar to those of wild ginseng [16].

Wild ginseng can grow in natural environments for decades or even hundreds of years and rarely become sick. In contrast, the survival rate of farmland cultivated ginseng seedlings was less than $25 \%$ after 3 years [17]. Farmland cultivated roots are harvested 5-6 years after planting, which may be related to the susceptibility of cultivated ginseng to disease. Farmland cultivated ginseng is susceptible to various soilborne diseases, among which root rot and root rust are the two most common diseases $[18,19]$. A previous study has shown that the Fusarium was a potential phytopathogen that can cause root rot [20]. Rhizosphere bacteria, belonging to Chloroflexi and Nitrospirae were confirmed to cause rusty root [19]. These diseases significantly hinder the development of the ginseng industry [21]. Hence, the study of rhizosphere microorganisms of ginseng is very important for the healthy growth and industrial development of ginseng.

Bacterial and fungal community changes during ginseng cultivation had received much attention. It has been shown that rhizosphere microbial community is affected by cultivation ages, developmental stages and cultivation modes [17, 22-24]. However, these studies mainly focused on cultivated ginseng [17]. Whereas is not clear the structure in the rhizosphere microbial communities of wild ginseng. We hypothesized that (1) the diversity and structure of the bacterial and fungal communities of the different type of ginseng rhizospheres are different (2) the pathogenicity and survival time of different ginseng may be related to the abundance of some rhizosphere microbial taxa.

To our knowledge, no study has investigated the contributions of different ginseng type to shaping the rhizosphere microbial community. Therefore, to explore the rhizosphere microbial community of understory wild ginseng, farmland cultivated ginseng and wild ginseng by 16S rRNA gene and internal transcribed spacer (ITS) region. The objective was to explore the compositions and diversity in rhizosphere bacterial and fungal communities associated with understory ginseng, wild ginseng and 
cultivated ginseng. The study also aimed to detect the presence of known pathogens of ginseng and compare their relative abundance among three types of ginsengs, with the goal of providing insights into the underlying mechanisms of ginseng planting and disease resistance.

\section{Results}

\section{Factors driving microbial communities in ginseng cultivars}

We studied the influence of soil physical and chemical properties and type on the microbial community. Mantel test showed that bacterial and fungal community composition were not significantly correlated with soil physical and chemical properties (Supplementary Table S1). However, the PERMANOVA result suggested type of ginsengs explained $90.118 \%$ and $84.699 \%$ of variance in bacteria and fungi, respectively (Table 3). The PCoA of Bray-Curtis distance matrix demonstrated that samples from the three groups showed clear separation, suggesting that the bacterial and fungal communities were obviously different among three types of ginsengs (Supplementary Figure S1).

\section{Rhizosphere community diversity in wild ginseng and cultivated ginseng}

We analyzed the bacterial and fungal communities from the rhizosphere soil in three types of ginsengs. We obtained 1, 135, 354 and 797, 696 total high-quality paired reads, which resulted in 4, 381 and 2, 679 OTUs for the bacterial and fungal data sets, respectively. The number of OTUs from LXG, CDG and WDG were 3,$183 ; 1,460 ; 3,000$ and $747 ; 922 ; 1,654$ in the bacterial and fungal community, respectively (Figure 1). The percentage of shared OTUs between LXG and WDG was smaller for the fungal community than for the bacterial community, and this pattern also occurred between LXG and CDG and between WDG and CDG. We also found that WDG shared more bacterial and fungal OTUs with LXG, and LXG and CDG shared the least fungal OUTs (Figure 1).

The rhizosphere microbial diversity differed among three type of ginsengs (Figure 2). The bacterial alpha diversity of $L X G$ and WDG were similar, however, that of CDG was significantly lower than those of YSWDG $(p<0.01)$ (Figure 2A-C). YSWDG had the highest fungal species richness (Chao 1), however, the fungal species evenness (Pielou) and fungal species diversity (Shannon) of WDG was the lowest ( $p<$ 0.01) (Figure 2D-F). The fungal alpha diversity of LXG was higher than CDG (Figure 2D-F).

\section{Bacterial and fungal community composition}

The composition and abundance for each taxon were obtained based on the OTU classification results. For bacteria, the dominant phyla were Proteobacteria, Acidobacteria, Actinobacteria and Chloroflexi in LXG (relative abundances of $30.76 \%, 27.92 \%, 8.51 \%$ and $4.57 \%$, respectively) and WDG (relative abundances of $32.61 \%, 25.39 \%, 11.92 \%$ and $8.39 \%$, respectively) (Figure 3A), However, Actinobacteria 
(23.75\%) was the most phylum in CDG, followed by Chloroflexi (21.85\%), Firmicutes (17.40\%) and Proteobacteria (13.53\%). The ANOVA analysis suggested that the proportions of each main four phyla were not significantly different between LXG and WDG, however, these four phyla (Proteobacteria, Acidobacteria, Actinobacteria and Chloroflexi) were significantly different in groups WDG and CDG, LXG and CDG, respectively. (Supplementary Figure S2, $p<0.01$ ). The most abundant bacterial classes were Alphaproteobacteria and Betaproteobacteria in LXG (relative abundances of $14.71 \%$ and $7.07 \%$, respectively) and WDG (relative abundances of $14.02 \%$ and $8.76 \%$, respectively), then Saprospirae (6.94\%) and Acidobacteria (6.94\%) in LXG, after Chloracidobacteria (12.02\%) and Deltaproteobacteria (7.17\%) in WDG. Ktedonobacteria (20.24\%), Bacilli (13.80\%), Actinobacteria (11.18\%) and Thermoleophilia (11.05\%) had the highest relative abundances in CDG (Figure 3B).

For fungi, Basidiomycota and Ascomycota accounted for more than $90 \%$ of the total abundance across all groups (Figure $3 C$ ), but the result of ANOVA indicated that the proportions of each phylum were different in each ginseng group (Supplementary Figure $S 3, p<0.01$ ). At the class level, the dominant classes were Agaricomycetes, class incertae_sedis and Leotiomycetes in LXG (relative abundances of $82.56 \%, 5.61 \%$ and $3.04 \%$, respectively) and WDG (relative abundances of $63.85 \%, 7.45 \%$ and $6.78 \%$ ) (Figure 3D), respectively. In CDG, the relative abundance of Sordariomycetes (45.92\%) was the highest, followed by Dothideomycetes (27.00\%) and Microbotryomycetes (7.22\%) (Figure 3D). At the genus level, the most abundant genus was Fusarium (28.37\%) in CDG; however, it was rare in LXG $(<0.01 \%)$ and WDG $(0.01 \%)$ (Figure 4A), and ANOVA suggested that the abundance of Fusarium was the highest in CDG ( $p<$ 0.01 ) (Figure $4 \mathrm{~B}$ ). Alternaria is a pathogenic fungi associated with ginseng rusty roots [25]. And the abundance of Alternaria in CDG than in LXG and WDG $(p<0.01)$ (Figure $4 C)$.

The LEfSe analysis of the bacterial communities showed that there were 68 differentially abundant taxa among the three groups of ginsengs. Of the 68 taxa, 23 were differentially abundant in WDG (Figure 5A, Supplementary Figure S4A), namely the Verrucomicrobia phylum and the Deltaproteobacteria, Betaproteobacteria, Acidobacteris_6, Chloracidobacteria and Anaerolineae classes. The enriched taxa in LXG were the phyla Bacteroidetes and Acidobacteria and the class Alphaproteobacteria. The differentially abundant taxa in the rhizosphere soils of CDG were the Firmicutes, Actinobacteria, WPS-2 and Chloroflexi phyla and the Gammaproteobacteria class.

The LEfSe analysis of the fungal communities from LXG, WDG and CDG showed that meanwhile, there were 69 differentially abundant taxa with an LDA score higher than 2.0 (Figure 5B, Supplementary Figure S4B). Among the 69 fungal taxa, 15 fungal taxa were differentially abundant in WDG, principally including the Mortierellomycota phylum, the Leotiomycetes and Eurotiomycetes classes, and the Russulales order. The abundant taxa in the rhizosphere soils of LXG were the Agaricomycetes and Tremellomycetes classes. The most differentially abundant fungal taxa were the Sordariomycetes, Dothideomycetes and Microbotryomycetes classes in CDG. These results indicated that Verrucomicrobia and Mortierellomycota phyla were significantly enriched in WDG, moreover, Alphaproteobacteria and Alphaproteobacteria classes were significantly enriched in LXG, in addition, The differentially abundant taxa in CDG were Chloroflexi phyla and Dothideomycetes classes. 


\section{Discussion}

\section{The type of ginseng obviously affected the rhizosphere microbial community}

We investigated the rhizosphere bacterial and fungal communities under three types of ginsengs, including understory wild ginseng, farmland cultivated ginseng and wild ginseng. The physical and chemical properties of soil in three types are different. In our study, Mantel test suggested soil physical and chemical properties had little effect on bacterial and fungal communities. In addition, PERMANOVA result indicated that the ginseng types significantly affected the rhizosphere microbial community. Previous studies showed that plant species or genotype had measurable effect their rhizosphere microbial community [26]. Therefore, we inferred that soil physical and chemical properties had little effect on bacterial and fungal communities. However, the type of ginseng obviously affected the rhizosphere microbial community. Different plant may form different microbial communities through interactions between plant roots and microorganisms $[27,28]$.

The microbial community is not only affected by host plant, but also affected by factors such as geographic location, climate et al, also other soil legacies (effect of soil management practices, effect of composition of the current/previous plant community) [27, 29-33]. In our study, we found that the proportion of shared fungal OTUs was much smaller than that of bacterial OTUs between any two pairs of three groups. Furthermore, WDG shared more fungal OTUs with LXG, and LXG and CDG shared the least fungal OTU. In addition, the distance between the two sampling locations of WDG and LXG was the closest, and the distance between the two sampling locations of LXG and CDG was the furthest. Hence, we inferred fungal communities appeared to be more affected by geographic distance than bacterial communities. The fungal community seem to be differentiate in geographic distance than are bacterial community $[34,35]$. The limited dispersal capacity of fungal communities might be the cause of this phenomenon [36]. Coleman-Derr et al (2016) also suggested that fungal communities were perhaps more shaped by geographic distance than bacterial communities in rhizosphere soils of Agave from California and Mexico [29]. Our analyses and comparisons across different sampling sites further suggested that geography plays a greater role in driving fungal than bacterial communities.

\section{Different bacterial diversity and fungal diversity in three types of ginsengs}

The bacterial alpha diversity of LXG and WDG were similar, and WDG shared more bacterial OTUs with LXG than with CDG. Maybe the understory wild ginseng is a semi-wild ginseng and its morphology and intrinsic quality of roots are similar to those of wild ginseng. Meanwhile, understory wild ginseng and wild ginseng both grow in mixed forests and deciduous broad-leaved forests, and there were similar vegetation types under the forest. These factors may lead to the similar bacterial diversity in understory wild ginseng and wild ginseng. However, analysis of the bacterial alpha diversity revealed a decreasing 
trend in CDG compared with WDG, showing a loss of natural bacterial diversity in the rhizosphere. Similarly, a lower in bacterial diversity was observed in cultivated maize as compared to its ancestors, and the Shannon index was higher for ancestors than for one cultivated maize [37]. A number of agronomic management practices may potentially influence bacterial diversity in cultivated ginseng. First, bacterial diversity would decrease over longer cultivation periods. This may be because older roots secrete low amounts of organic matter, or because long-term cultivation depletes nutrient cycling is altered in cultivated soils $[38,39]$. Second, the growth environments of the cultivated ginseng and the wild ginseng were very different, and environmental factors such as climate and annual precipitation influence the diversity of rhizosphere microorganisms [40]. Third, pesticide disposal also affects rhizosphere microorganisms and reduces the microbial diversity of cultivated ginseng. Pesticides were applied to reduce pests and diseases and may have indirectly affected root exudates or directly affected the diversity of rhizosphere microorganisms during ginseng cultivation [41, 42]. In addition, the decrease in bacterial diversity may occur because domesticated crops and wild crops may interact with rhizosphere microbial communities differently [43]. The roots of farmland cultivated ginseng grow more quickly than those of wild ginseng, and roots may secrete different types of organic compounds, which can have an important impact on below-ground microbial communities. For instance, previous studies have shown that plants growing at a faster growth rate might excrete compounds into the rhizosphere soil that result in reduced microbial diversity [44-46].

In contrast, fungal diversity showed the opposite phenomenon. We found that the Chao 1 was the highest in WDG, which may be associated with the greater number of fungal OTUs in WDG than in CDG and LXG. In addition, the fungal diversity (Shannon and Pielou) was the lowest in WDG. A similar phenomenon has been observed in soybean and their wild species, and the fungal diversity of cultivated soybean increased compared to its wild type [47]. Previous studies suggested that the death rate of $P$. notoginseng was significantly negatively correlated with fungal diversity, indicating that fungal diversity was a potential indicator of soil health [48]. Moreover, the fungal diversity in cultivated ginseng was higher than that in wild ginseng, which might be the result of plant domestication leading to the prevalence of pathogens [49]. This may be related to the low survival rate of cultivated ginseng and its susceptibility to disease. Furthermore, the fungal alpha diversity of LXG was higher than CDG, which may might be related to agricultural management for understory wild ginseng. Wild ginseng and farmland cultivated ginseng were planted directly in the field, but understory wild ginseng seedlings were grown for several years before transplanting them to another forest. The rhizosphere microbial community may be assembled early in plant development, and later transplantation may alter the diversity of the microbial community [29]. Therefore, the rhizosphere microbial diversity may be affected by the method of agricultural management.

\section{Changes in the composition of microbial communities among the three types of ginsengs}


In the composition of bacterial communities, our study indicated that Proteobacteria and Acidobacteria all existed in WDG, LXG and CDG, and previous studies confirmed that Proteobacteria and Acidobacteria were the dominant populations in the rhizosphere soil of ginseng [50]. The relative abundance of Chloroflexi in CDG was higher than that in WDG and LXG $(p<0.01)$. [19] suggested that root rust may be caused by Chloroflexi in rhizosphere microbial communities based on five cultivated ginseng samples with different severity of rusty root disease. Although the rhizosphere soils in our study came from healthy ginseng, we also inferred Chloroflexi contain species that are pathogenic to ginseng. In addition, Verrucomicrobia was significantly high abundance in WDG and also has been found in the rhizosphere of the common bean, and Verrucomicrobia was also mainly found in wild bean accessions [43].

Verrucomicrobia might have a special affinity for wild species. These phyla need further reseach in the future.

In fungi community, the same fungal phyla were detected but the fungal community composition, also at phylum level, the relatively abundances of Basidiomycota and Ascomycota was different in LXG, CDG and WDG. In an earlier study, Ascomycota, Basidiomycota and were the dominant phyla during the continuous cropping of $P$. notoginseng, but their relative abundances relative abundances [37]. Ascomycota, which has an important role in the decomposition of soil organic matter and largely dominates the active fungal community through the assimilation of root exudates [51]. Furthermore, the main pathogenic fungus Fusarium that caused root rot belongs to the Ascomycota, which was the most predominant phylum in CDG. Fusarium is a potential phytopathogen (includes species that are pathogenic (but also species that are not patogenic) that can cause root rot in various species, including ginseng, American ginseng, soybean and sunflower [52-55]. We found that the abundance of Fusarium was the highest in CDG, but only $0.01 \%$ in WDG $(p<0.01)$. Likewise, cultivated rice had a higher abundance of pathogens comparing with the wild varieties [47]. Moreover, Alternaria is a pathogenic fungi associated with ginseng rusty roots [25]. And the abundance of Alternaria in CDG than in LXG and WDG. Our finding was consistent with the results of other studies, further suggesting that plant domestication may have stimulated the epidemic of pathogens and affect the ability of modern crops to establish beneficial relationships with the rhizosphere [43]. This also explains how wild ginseng can grow in natural environments for decades or even hundreds of years and rarely become sick. In addition, continuous planting may also lead to an increase in pathogenic microorganisms in the rhizosphere. The pathogenic microbes, including Alternaria and Fusarium, that were highly enriched in 30-year continuous sugar beet cropping [56].

\section{Methods}

\section{Sampling Sites and Samples Collection}

Rhizosphere soil samples were collected from three type of ginsengs, including understory wild ginseng (the seedlings of the cultivated ginseng were planted directly into the soil and no additional fertilizers or pesticides were applied to the soil during the growth of the ginseng), farmland cultivated ginseng (seeds of the cultivated ginseng were planted in farmland that was once forested, and it is sprayed with 
pesticides during growth) and wild ginseng (wild ginseng grows naturally in the forest). All ginseng was grown for about 15 years. wild ginseng and understory wild ginseng both grow in mixed forests and deciduous broad-leaved forests, and there were similar vegetation types under the forest. The rhizosphere samples of understory wild ginseng were collected from Linjiang city of Jilin Province, and the soil was Mollic Albi-boric Cambosols (sand 51\%, silt 32\%, clay 17\%) with PH (5.99), then chemical characteristics of the soil were $82 \mathrm{mg} \mathrm{P} \mathrm{kg-1,} 199 \mathrm{mg} \mathrm{N} \mathrm{kg-1}$ and $2198 \mathrm{mg} \mathrm{K} \mathrm{kg-1}$ with PH (5.99). The soil samples of farmland cultivated ginseng were from Ji'an city of Jilin Province, and the soil was Mollic bori-Udic Cambosols (sand 33\%, silt 49\%, clay 18\%), and chemical characteristics of the soil were (mg kg-1): 146 mg P kg-1, 401 mg N kg-1 and 1823 mg K kg-1 with PH (6.62). The rhizosphere soils of wild ginseng were collected from Korean autonomous county, Jilin Province. The soil was Mollic bori-Udic Cambosols (sand $45 \%$, silt $37 \%$, clay $18 \%$ ), and chemical characteristics of the soil were (mg kg-1): $146 \mathrm{mg} \mathrm{P} \mathrm{kg}-1,401 \mathrm{mg}$ $\mathrm{N} \mathrm{kg-1}$ and $1823 \mathrm{mg} \mathrm{K} \mathrm{kg-1}$ with PH (5.99) (Table 1, Table 2). The soil characteries were mapped using the National Earth System Science Data Center [57].

In this experiment, three groups understory wild ginseng, farmland cultivated ginseng and wild ginseng were defined. Groups LXG, CDG and WDG represent the rhizosphere soils of understory wild ginseng, farmland cultivated ginseng and wild ginseng, respectively. All rhizosphere soil samples were collected in August 2018. A collection of ginseng rhizosphere soil was made, and samples were taken from a depth of $20 \mathrm{~cm}$ using a sterile shovel. Ginseng plants were carefully removed from the ground, keeping the root system intact. The large clumps of soil on the roots were removed, then brushed soil attached to the roots with a brush. Each soil samples were passed through a $2 \mathrm{~mm}$ sieve, finally into a sterile tube. The soil samples in each growth state are from at least three healthy, disease-free roots of ginseng (one to three rhizosphere soil samples were collected from the roots of each ginseng). In total, the rhizosphere soils samples of DXSLX, PHTS and YSWDG were set up with seven, five and six, respectively (Table 1). All samples were then transported to the liquid nitrogen within one hour and immediately transported to the laboratory. Finally, the soil samples were stored at $-80^{\circ} \mathrm{C}$ until genomic DNA extraction using an E.Z.N.A.® Stool DNA Kit (Omega, Shanghai).

\section{PCR, amplicon quantification, HiSeq library construction and sequencing}

The variable V3-V4 region of the bacterial 16S rRNA gene and fungal ITS1 region were amplified from each sample with the primers pairs 341F (5'-ACTCCTACGGGAGGCAGCAG-3') / 806R (5'GGACTACHVGGGTWTCTAAT-3') and ITS-1 (5'-CTTGGTCATTTAGAGGAAGTAA-3') / ITS-2 (5'GCTGCGTTCTTCATCGATGC-3') [44, 58]. All PCRs reactions were performed using NEB Phusion HighFidelity PCR Master Mix following the manufacturer's recommendations. The PCR contained $30 \mathrm{ng}$ of DNA, $4 \mu \mathrm{L}$ of PCR primer mix and $25 \mu \mathrm{L}$ of PCR Master Mix. The following PCR conditions were used for 16S rRNA and ITS: $98^{\circ} \mathrm{C}$ for 3 min; followed by 30 cycles of $98^{\circ} \mathrm{C}$ for $45 \mathrm{~s}, 55^{\circ} \mathrm{C}$ for $45 \mathrm{~s}$ and $72{ }^{\circ} \mathrm{C}$ for 45 $\mathrm{s}$; and a final extension of $72{ }^{\circ} \mathrm{C}$ for $7 \mathrm{~min}$. Then, the PCR products integrity was tested by $1 \%$ agarose gel electrophoresis and purified using Ampure XP beads (Beckman, America) to remove the unspecific 
products. The final library was quantitated in two ways: determination of the average molecule length using an Agilent 2100 bioanalyzer instrument (Agilent DNA 1000 reagents, America), and quantification of the library by real-time quantitative PCR. The qualified libraries were sequenced pair-end on the system with the sequencing strategy PE250 under the HiSeq platform (Illumina, America).

\section{Data analysis and statistics}

After removing the barcode and primer sequences, the remaining reads were merged based on overlapping regions using FLASH (fast length adjustment of short reads, v1.2.11) within paired-end reads [59]. Reads with ambiguous bases, an average Phred score less than 20 and the length lower than $10 \mathrm{bp}$ were removed. Then, operational taxonomic units (OTUs) were clustered with a $97 \%$ similarity cut off by using UPARSE (version 7.0.1090) [60]. The chimeric sequences were identified and removed using UCHIME software (v4.2.40) [61]. OTUs undoubtedly belonging to chloroplasts or mitochondria were also removed. Subsequently, the taxonomic classification of the representative sequence for each bacterial and fungal OTUs was annotated using Greengenes (v201304) and UNITE (Version 7.2) reference databases, respectively, with the RDP Classifier v2.2 (Ribosomal Database Project). The significant difference in rhizosphere microbe community composition among three type of ginsengs was evidenced by ANOVA (One-way Analysis of Variance, $p<0.05$ ) in SPSS_Statistics_23. A venn plotter was used to obtain the number of unique and common OTUs common to distinct types of ginsengs using the 'VennDiagram' R package ( $v$ 3.1.1). The alpha diversity of the bacterial and fungal communities was calculated with the Chao 1 (species richness), Pielou (species evenness) and Shannon (species diversity) indexes for each group of ginseng using in MOTHUR (v1.31.2) [62]. The Chao1, Pielou and Shannon indexes were calculated. The differences in alpha diversity were determined by Tukey's honestly significant difference test in R package ( $v$ 3.1.1) $(p<0.05)$. Mantel tests and Permutational ANOVAS (PERMANOVA) were performed to assess the correlation between rhizosphere microbial communities and soil physical and chemical properties, type of ginseng by using R package 'vegan' ( $v$ 2.5-7), respectively [63]. Principal coordinate analysis (PCoA) was performed in QIIME software ( $v 1.80)$ to reflect the beta diversity of the microbial community, evaluate the similarity in community composition among the different groups of ginseng based on the Bray-Curtis distance matrix [64]. Linear discriminant analysis (LDA) effect sizes (LEfSe) was used to detect notably different taxa among the samples using the Galaxy online analytics platform, and LEfSe identity different abundant taxa with an linear discriminant analysis (LDA) score higher than 2.0 (http://huttenhower.sph.havard.edu/galaxy).

\section{Conclusions}

Our study confirmed that there was no obvious correlation between rhizosphere microorganisms and soil physical and chemical properties, but the type of ginseng obviously affected the rhizosphere microbial community. By comparing the rhizosphere microbial community of understory wild ginseng, wild ginseng and farmland cultivated ginseng, the result suggested the fungal communities were perhaps more shaped than bacterial communities. We also found lower bacterial diversity and higher fungal diversity in 
CDG compared with WDG. Domestication might be a main factor affecting the composition and diversity of the rhizosphere communities. Furthermore, we detected the presence of known pathogens Chloroflexi, Alternaria and Fusarium were potential phytopathogens in farmland cultivated ginseng, this can explain the fact that farmland cultivated ginseng is more susceptible to disease than wild ginseng. This result might provide insights into the underlying mechanisms of ginseng planting and actionable information for soil management. We can cultivate ginseng by simulating the rhizosphere microorganism of wild ginseng, which can reduce the prevalence of cultivated ginseng.

\section{Declarations}

\section{Conflict of Interest}

The authors declare that the research was conducted in the absence of any commercial or financial relationships that could be construed as a potential conflict of interest.

\section{Author Contributions}

M. S. and X. F. designed the experiments. X. F. and H. W. performed most of experiments and analyzed the data. Other authors assisted in experiments and discussed the results. X. F. and H. W. wrote the manuscript.

\section{Funding}

This work was supported by National Natural Science Foundation of China (grant numbers 31770243).

\section{Acknowledgments}

We thank reviewers and editors for their constructive comments that greatly improved our manuscript. We also thank Dr. Hongxing Xiao and Wei Zhang for helping to collected the samples in this study.

\section{Availability of data and materials}

The sequencing dataset analyzed during the current study is available in the NCBI Sequence Read Archive (PRJNA701796 and PRJNA701800).

\section{References}

1. Lugtenberg B, Kamilova F: Plant-growth-promoting rhizobacteria. In: Annual Review of Microbiology. vol. 63; 2009: 541-556. 
2. Berendsen RL, Pieterse CMJ, Bakker PAHM: The rhizosphere microbiome and plant health. TRENDS PLANT SCI 2012, 17(8):478-486.

3. Turner TR, James EK, Poole PS: The plant microbiome. Genome Biology 2013, 14(6).

4. Vacheron J, Desbrosses G, Bouffaud ML, Touraine B, Moënne-Loccoz Y, Muller D, Legendre L, Wisniewski-Dyé F, Prigent-Combaret C: Plant growth-promoting rhizobacteria and root system functioning. Frontiers in Plant Science 2013, 4(SEP).

5. Philippot L, Raaijmakers JM, Lemanceau P, Van Der Putten WH: Going back to the roots: The microbial ecology of the rhizosphere. Nature Reviews Microbiology 2013, 11(11):789-799.

6. Badri DV, Vivanco JM: Regulation and function of root exudates. Plant, Cell and Environment 2009, 32(6):666-681.

7. Rengel Z, Marschner P: Nutrient availability and management in the rhizosphere: Exploiting genotypic differences. New Phytol 2005, 168(2):305-312.

8. Somers E, Vanderleyden J, Srinivasan M: Rhizosphere bacterial signalling: A love parade beneath our feet. Crit Rev Microbiol 2004, 30(4):205-240.

9. Emmert EAB, Geleta SB, Rose CM, Seho-Ahiable GE, Hawkins AE, Baker KT, Evans AS, Harris ME, Mrozinski AC, Folkoff ME et al: Effect of land use changes on soil microbial enzymatic activity and soil microbial community composition on Maryland's Eastern Shore. Applied Soil Ecology 2021, 161.

10. Yesilonis I, Szlavecz K, Pouyat R, Whigham D, Xia L: Historical land use and stand age effects on forest soil properties in the Mid-Atlantic US. Forest Ecology and Management 2016, 370:83-92.

11. Ernst E: Panax ginseng: An overview of the clinical evidence. Journal of Ginseng Research 2010, 34(4):259-263.

12. Baeg IH, So SH: The world ginseng market and the ginseng (Korea). Journal of Ginseng Research 2013, 37(1):1-7.

13. Wu H, Yang HY, You XL, Li YH: Diversity of endophytic fungi from roots of Panax ginseng and their saponin yield capacities. SpringerPlus 2013, 2(1):1-9.

14. Zhao J, Q.: Analysis of amino acid composition and total content of the ginsenoside in wild and cultivated ginseng. ACTA SCIENTIARUM NATURALIUM UNIV ERSI TATIS JILINENSIS 1989(03):99-101.

15. Li MR, Shi FX, Zhou YX, Li YL, Wang XF, Zhang C, Wang XT, Liu B, Xiao HX, Li LF: Genetic and Epigenetic Diversities Shed Light on Domestication of Cultivated Ginseng (Panax ginseng). Molecular Plant 2015, 8(11):1612-1622. 
16. Sun H, Wang Q, Zhang Y, Yang Z, Xu C: Integrated Evaluation of Soil Fertility of $\triangle P$ Panax ginseng under Different Cultivation Modes. Journal of Jilin Agricultural University 2015, 37(03):323-331.

17. Ying YX, Ding WL, Li Y: Characterization of soil bacterial communities in rhizospheric and nonrhizospheric soil of panax ginseng. Biochemical Genetics 2012, 50(11-12):848-859.

18. Chanyong L, Kim KY, Lee JE, Kim S, Ryu D, Choi JE, An G: Enzymes hydrolyzing structural components and ferrous ion cause rusty-root symptom on ginseng (Panax ginseng). Journal of Microbiology and Biotechnology 2011, 21(2):192-196.

19. Wang Q, Sun H, Xu C, Ma L, Li M, Shao C, Guan Y, Liu N, Liu Z, Zhang S et al: Analysis of rhizosphere bacterial and fungal communities associated with rusty root disease of Panax ginseng. Applied Soil Ecology 2019, 138:245-252.

20. Miao ZQ, Li SD, Liu X, Chen YJ, Li YH, Wang Y, Guo RJ, Xia ZY, Zhang KQ: The causal microorganisms of Panax notoginseng root rot disease. Scientia Agricultura Sinica 2006, 39:1371-1378.

21. Rahman M, Punja ZK: Biochemistry of ginseng root tissues affected by rusty root symptoms. Plant Physiology and Biochemistry 2005, 43(12):1103-1114.

22. Xiao C, Yang L, Zhang L, Liu C, Han M: Effects of cultivation ages and modes on microbial diversity in the rhizosphere soil of panax ginseng. Journal of Ginseng Research 2016, 40(1):28-37.

23. Dong L, Xu J, Li Y, Fang H, Niu W, Li X, Zhang Y, Ding W, Chen S: Manipulation of microbial community in the rhizosphere alleviates the replanting issues in Panax ginseng. Soil Biol Biochem 2018, 125:64-74.

24. Dong L, Xu J, Zhang L, Cheng R, Wei G, Su H, Yang J, Qian J, Xu R, Chen S: Rhizospheric microbial communities are driven by Panax ginseng at different growth stages and biocontrol bacteria alleviates replanting mortality. Acta Pharmaceutica Sinica B 2018, 8(2):272-282.

25. Wei X, Wang X, Cao P, Gao Z, Chen AJ, Han J: Microbial Community Changes in the Rhizosphere Soil of Healthy and Rusty <i>Panax ginseng $</ i>$ and Discovery of Pivotal Fungal Genera Associated with Rusty Roots. BioMed Research International 2020, 2020:8018525.

26. Hernandez-Teran A, Navarro-Diaz M, Benitez M, Lira R, Wegier A, Escalante AE: Host genotype explains rhizospheric microbial community composition: The case of wild cotton metapopulations (Gossypium hirsutum L.) in Mexico. FEMS Microbiology Ecology 2020, 96(8).

27. Berg G, Smalla K: Plant species and soil type cooperatively shape the structure and function of microbial communities in the rhizosphere. FEMS Microbiology Ecology 2009, 68(1):1-13.

28. Wang XB, Hsu CM, Dubeux JCB, Mackowiak C, Blount A, Han XG, Liao HL: Effects of rhizoma peanut cultivars (Arachis glabrata Benth.) on the soil bacterial diversity and predicted function in nitrogen 
fixation. ECOL EVOL 2019, 9(22):12676-12687.

29. Coleman-Derr D, Desgarennes D, Fonseca-Garcia C, Gross S, Clingenpeel S, Woyke T, North G, Visel A, Partida-Martinez LP, Tringe SG: Plant compartment and biogeography affect microbiome composition in cultivated and native Agave species. New Phytologist 2016, 209(2):798-811.

30. Qaderi MM, Reid DM, Yeung EC: Morphological and physiological responses of canola (Brassica napus) siliquas and seeds to UVB and $\mathrm{CO}<\mathrm{inf}>2</ \mathrm{inf}>$ under controlled environment conditions. Environmental and Experimental Botany 2007, 60(3):428-437.

31. Smith CR, Blair PL, Boyd C, Cody B, Hazel A, Hedrick A, Kathuria H, Khurana P, Kramer B, Muterspaw $\mathrm{K}$ et al: Microbial community responses to soil tillage and crop rotation in a corn/soybean agroecosystem. Ecology and Evolution 2016, 6(22):8075-8084.

32. Soman C, Li D, Wander MM, Kent AD: Long-term fertilizer and crop-rotation treatments differentially affect soil bacterial community structure. Plant and Soil 2017, 413(1-2):145-159.

33. Zhang B, Liang $\mathrm{C}, \mathrm{He} \mathrm{H}$, Zhang $\mathrm{X}$ : Variations in Soil Microbial Communities and Residues Along an Altitude Gradient on the Northern Slope of Changbai Mountain, China. PLOS ONE2013, 8(6).

34. Meiser A, Bálint M, Schmitt I: Meta-analysis of deep-sequenced fungal communities indicates limited taxon sharing between studies and the presence of biogeographic patterns. New Phytologist 2014, 201(2):623-635.

35. Shakya M, Gottel N, Castro H, Yang ZK, Gunter L, Labbé J, Muchero W, Bonito G, Vilgalys R, Tuskan $\mathrm{G}$ et al: A Multifactor Analysis of Fungal and Bacterial Community Structure in the Root Microbiome of Mature Populus deltoides Trees. PLOS ONE 2013, 8(10).

36. Taylor JW, Turner E, Townsend JP, Dettman JR, Jacobson D: Eukaryotic microbes, species recognition and the geographic limits of species: Examples from the kingdom Fungi. Philosophical Transactions of the Royal Society B: Biological Sciences 2006, 361(1475):1947-1963.

37. Szoboszlay M, Lambers J, Chappell J, Kupper JV, Moe LA, McNear DH: Comparison of root system architecture and rhizosphere microbial communities of Balsas teosinte and domesticated corn cultivars. Soil Biology and Biochemistry 2015, 80:34-44.

38. Li Y, Ying YX, Zhao DY, Ding WLJOJoG: Microbial community diversity analysis of Panax ginseng rhizosphere and non-rhizosphere soil using randomly amplified polymorphic DNA method. 2012, 2(2):95102.

39. Ying, Yi-Xin, Li, Yong, Ding, Wan-Long, Hu, Chen-Yun: Dynamics of Panax ginseng Rhizospheric Soil Microbial Community and Their Metabolic Function zicrobial Community and Their Metabolic Function. In: Cross-Strait and csnr National Chinese Medicine and Natural Medicine Resources Academic Seminar 2012. 
40. Wang X, Yu S, Zhou L, Fu S: Soil microbial characteristics and the influencing factors in subtropical forests. Shengtai Xuebao/ Acta Ecologica Sinica 2016, 36(1):8-15.

41. Chen S, Li X, Lavoie M, Jin Y, Xu J, Fu Z, Qian H: Diclofop-methyl affects microbial rhizosphere community and induces systemic acquired resistance in rice. Journal of Environmental Sciences (China) 2017, 51:352-360.

42. Arango L, Buddrus-Schiemann K, Opelt K, Lueders T, Haesler F, Schmid M, Ernst D, Hartmann A: Effects of glyphosate on the bacterial community associated with roots of transgenic Roundup Ready $B$ soybean. European Journal of Soil Biology 2014, 63:41-48.

43. Pérez-Jaramillo JE, Mendes R, Raaijmakers JM: Impact of plant domestication on rhizosphere microbiome assembly and functions. Plant Molecular Biology 2016, 90(6):635-644.

44. Haichar FEZ, Marol C, Berge O, Rangel-Castro JI, Prosser JI, Balesdent J, Heulin T, Achouak W: Plant host habitat and root exudates shape soil bacterial community structure. ISME J 2008, 2(12):12211230.

45. Oger PM, Mansouri H, Nesme X, Dessaux YJ: Engineering root exudation of Lotus towards the production of two novel carbon compounds leads to the selection of distinct microbial populations in the rhizosphere. Microbial Ecology 2004, 47(1):96-103.

46. Grayston SJ, Wang S, Campbell CD, Edwards AC: Selective influence of plant species on microbial diversity in the rhizosphere. Soil Biology and Biochemistry 1998, 30(3):369-378.

47. Shi S, Chang J, Tian L, Nasir F, Ji L, Li X, Tian C: Comparative analysis of the rizomicrobiome of the wild versus cultivated crop: insights from rice and soybean. Archives of Microbiology 2019, 201(7):879-888.

48. Dong $\mathrm{L}, \mathrm{Xu} \mathrm{J}$, Feng G, Li X, Chen S: Soil bacterial and fungal community dynamics in relation to Panax notoginseng death rate in a continuous cropping system. Scientific Reports 2016, 6.

49. Keesing F, Belden RK, Daszak R, Dobson R, Harvell RD, Holt RD, Hudson R, Jolles R, Jones RE, Mitchell REJN: Impacts of biodiversity on the emergence and transmission of infectious diseases. 2010, 468(7324):647-652.

50. Ying YX, Ding WL, Li Y: Characterization of soil bacterial communities in rhizospheric and nonrhizospheric soil of panax ginseng. Biochem Genet 2012, 50(11-12):848-859.

51. Voriskova J, Baldrian P: Fungal community on decomposing leaf litter undergoes rapid successional changes. ISME Journal 2013, 7(3):477-486.

52. Wei X, Wang X, Cao P, Gao Z, Chen AJ, Han J: Microbial Community Changes in the Rhizosphere Soil of Healthy and Rusty Panax ginseng and Discovery of Pivotal Fungal Genera Associated with Rusty 
Roots. BioMed Research International 2020, 2020.

53. Glinushkin AP, Ovsyankina AV, Kornyukov DA: Diagnosis of fungi of the genus Fusarium and Alternaria, Bipolaris, causing diseases of sunflower, and immunological methods for the evaluation and selection of genotypes to the pathogens. IOP Conference Series: Earth and Environmental Science 2021, 663(1):012049.

54. Okello PN, Petrovic K, Singh AK, Kontz B, Mathew FM: Characterization of species of Fusarium causing root rot of Soybean (Glycine max L.) in South Dakota, USA. Canadian Journal of Plant Pathology 2020, 42(4):560-571.

55. Punja ZK, Wan A, Goswami RS, Verma N, Rahman M, Barasubiye T, Seifert KA, Lévesque CA: Diversity of Fusarium species associated with discolored ginseng roots in British Columbia. Canadian Journal of Plant Pathology 2007, 29(4):340-353.

56. Huang W, Sun D, Fu J, Zhao H, Wang R, An Y: Effects of continuous sugar beet cropping on rhizospheric microbial communities. Genes 2020, 11(1).

57. National Earth System Science Data Center, . Soil SubCenter, National Science \& Technology Infrastructure of China. (http://soilgeodatacn) 2020.

58. Ullah A, Akbar A, Luo Q, Khan AH, Manghwar H, Shaban M, Yang X: Microbiome Diversity in Cotton Rhizosphere Under Normal and Drought Conditions. Microbial Ecology 2019, 77(2):429-439.

59. Magoč T, Salzberg SL: FLASH: Fast length adjustment of short reads to improve genome assemblies. Bioinformatics 2011, 27(21):2957-2963.

60. Edgar RC: UPARSE: Highly accurate OTU sequences from microbial amplicon reads. NAT METHODS 2013, 10(10):996-998.

61. Edgar RC, Haas BJ, Clemente JC, Quince C, Knight R: UCHIME improves sensitivity and speed of chimera detection. Bioinformatics 2011, 27(16):2194-2200.

62. Schloss PD, Westcott SL, Ryabin T, Hall JR, Hartmann M, Hollister EB, Lesniewski RA, Oakley BB, Parks DH, Robinson CJ et al: Introducing mothur: Open-source, platform-independent, communitysupported software for describing and comparing microbial communities. Applied and Environmental Microbiology 2009, 75(23):7537-7541.

63. Dixon P: VEGAN, a package of $\mathbf{R}$ functions for community ecology. Journal of Vegetation Science 2003, 14(6):927-930.

64. Caporaso JG, Kuczynski J, Stombaugh J, Bittinger K, Bushman FD, Costello EK, Fierer N, Pẽa AG, Goodrich JK, Gordon JI et al: QIIME allows analysis of high-throughput community sequencing data. NAT METHODS 2010, 7(5):335-336. 


\section{Tables}

Table 1. Geographic location, types of ginsengs and the number of rhizosphere soils samples in three types of ginsengs.

\begin{tabular}{|c|c|c|c|c|c|}
\hline & location & latitude & longitude & Growth status & $\mathrm{n}$ \\
\hline DXSLX & Linjiang country & $\begin{array}{l}\mathrm{N} 41^{\circ} 75 \\
36.86^{\prime \prime}\end{array}$ & $\begin{array}{l}\mathrm{E} 127^{\circ} 60 \\
29.35^{\prime \prime}\end{array}$ & $\begin{array}{l}\text { understory wild } \\
\text { ginseng }\end{array}$ & 7 \\
\hline PHTS & Ji'an city & $\begin{array}{l}\mathrm{N} 41^{\circ} 31 \\
\text { '54.46'" }\end{array}$ & $\begin{array}{l}\mathrm{E} 125^{\circ} 91 \\
\text { '48.33'" }\end{array}$ & cultivate ginseng & 5 \\
\hline YSWDG & $\begin{array}{l}\text { Korean Autonomous County of } \\
\text { Changbai }\end{array}$ & $\begin{array}{l}\mathrm{N} 41^{\circ} 31 \\
' 19.31^{\prime \prime}\end{array}$ & $\begin{array}{l}\mathrm{E} 127^{\circ} 57 \\
07.38^{\prime \prime}\end{array}$ & wild ginseng & 6 \\
\hline
\end{tabular}

Note: $n$, the number of rhizosphere soil samples.

Table 2. The physical and chemical properties of the soil in three types of ginsengs.

\begin{tabular}{|llll|}
\hline & DXSLX & PHTS & YSWDG \\
\hline $\begin{array}{l}\text { soil } \\
\text { taxonomy }\end{array}$ & $\begin{array}{l}\text { Mollic Albi-boric } \\
\text { Argosolos }\end{array}$ & $\begin{array}{l}\text { Mollic bori-Udic } \\
\text { Cambosols }\end{array}$ & $\begin{array}{l}\text { Mollic bori-Udic } \\
\text { Cambosols }\end{array}$ \\
\hline TP (mg kg-1) & 82 & 146 & 146 \\
\hline TN (mg kg-1) & 199 & 401 & 401 \\
\hline TK (mg kg-1) & 2198 & 1823 & 1823 \\
\hline PH & 5.99 & 6.62 & 5.99 \\
\hline Sand (\%) & 51 & 33 & 45 \\
\hline Silt (\%) & 32 & 49 & 37 \\
\hline Clay (\%) & 17 & 18 & 18 \\
\hline
\end{tabular}

Note: P, total phosphorus; $\mathrm{N}$, total nitrogen; $\mathrm{K}$, total potassium; Sand, the content of sand in soil; Silt, the content of silt in soil; Clay, the content of clay in soil.

Table 3. PERMANOVAs of the influence of ginseng types on microbial communities associated.

\begin{tabular}{|lllllll|}
\hline \multicolumn{3}{|c|}{ bacterial community } & \multicolumn{4}{c|}{ fungal community } \\
\hline factor & F & R2 & P & F & R2 & P \\
\hline types & 68.3980 & 0.90118 & $<0.01$ & 41.5160 & 0.84699 & $<0.01$ \\
\hline
\end{tabular}

Note: F, F.model; R2, Variation ; P, P-value. 
Figures

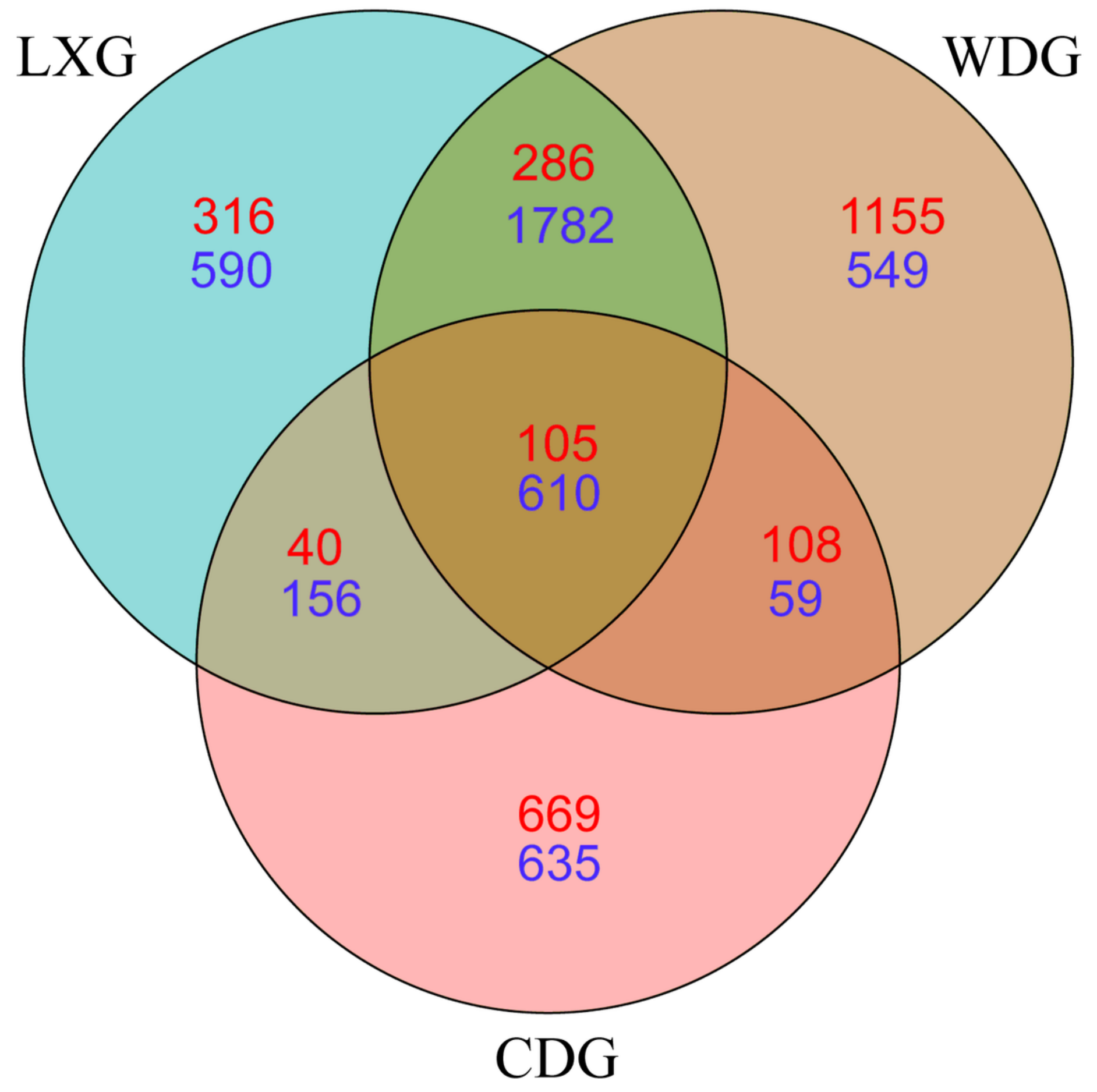

Figure 1

Venn diagrams of shared bacterial (blue) and fungal (red) OTUs in rhizosphere of three types of ginsengs. 
(A)

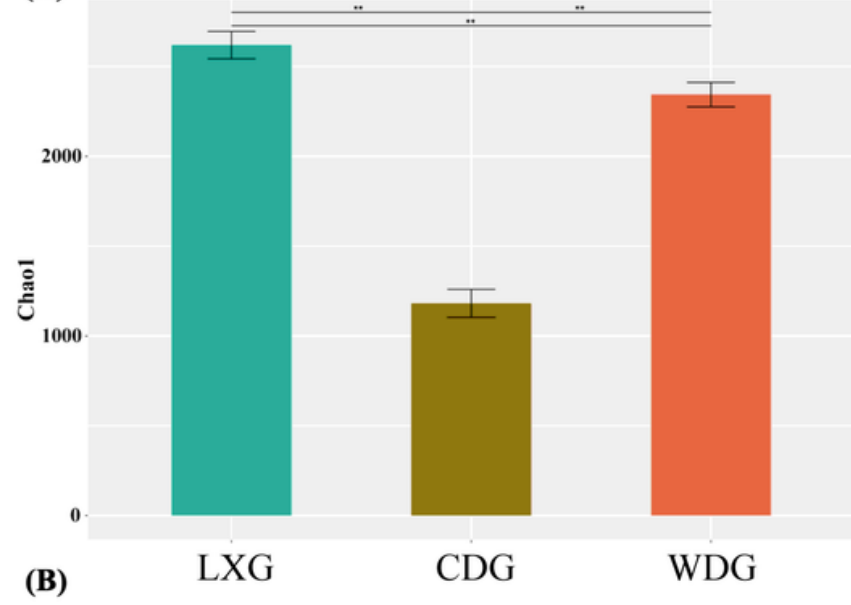

(B)

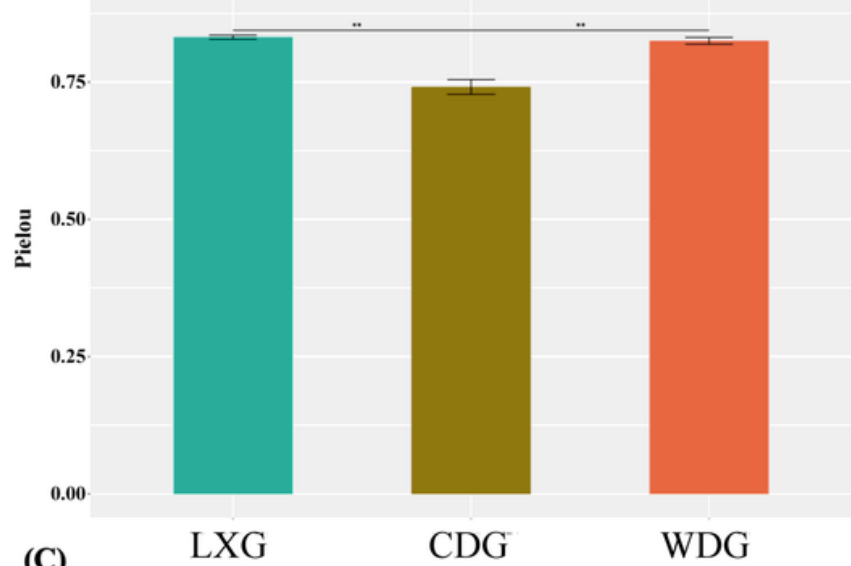

(C)

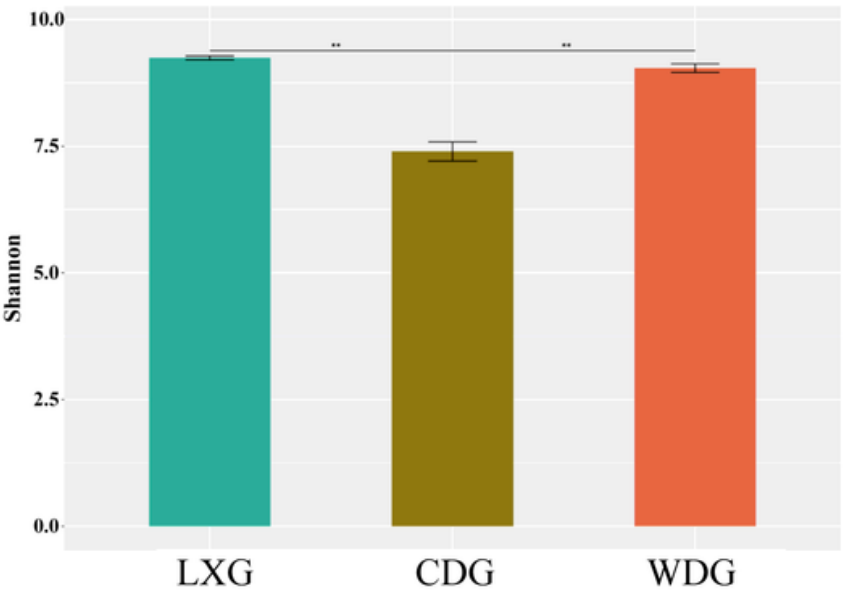

(D)
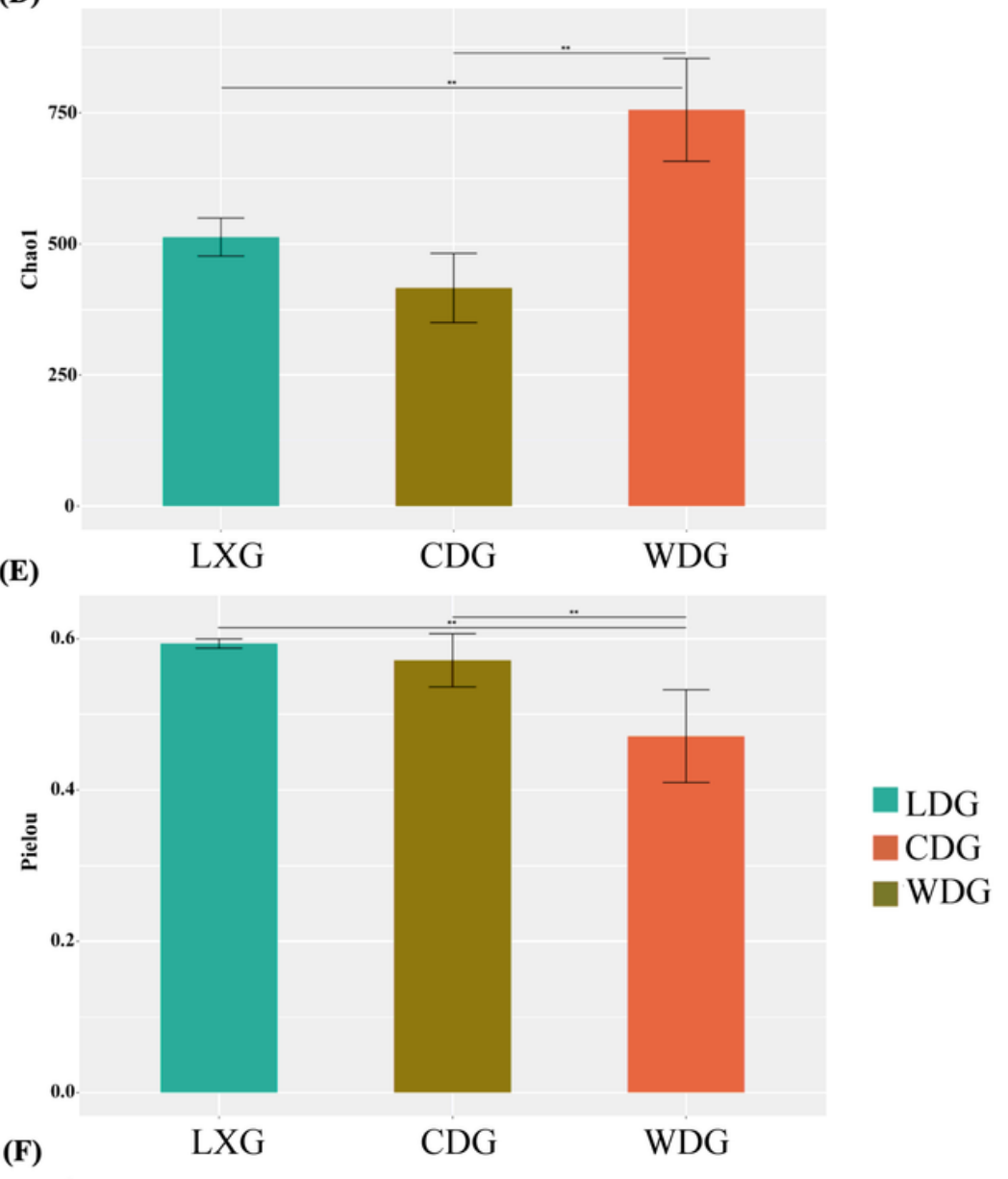

\section{Figure 2}

Chao 1, Pielou and Simpson indexes in the bacteria (A), (B), (C) and fungi (D), (E), (F) from three types of ginsengs in rhizosphere, data were means \pm standard error. *Significant at the 0.05 probability level. **Significant at the 0.01 probability level. 

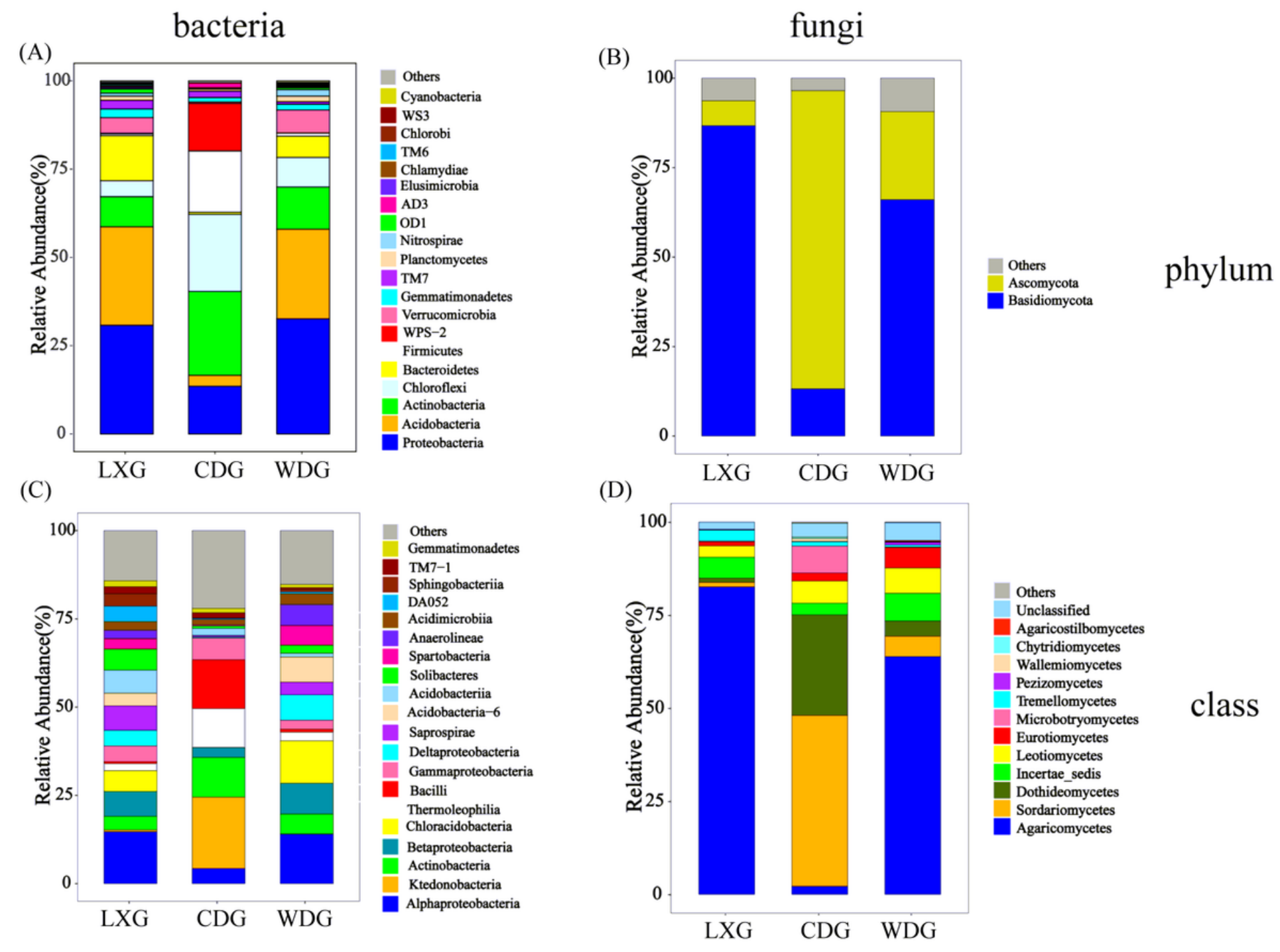

Figure 3

The composition of bacterial and fungal community from different types of ginseng rhizosphere. The phylum level of bacteria (A) and fungi (C), and the class level of bacteria (B) and fungi (D). The relative abundances in the top 20 were chosen to exhibit. Others represented of low relative abundance that ranks lower than top 20. 
(A)

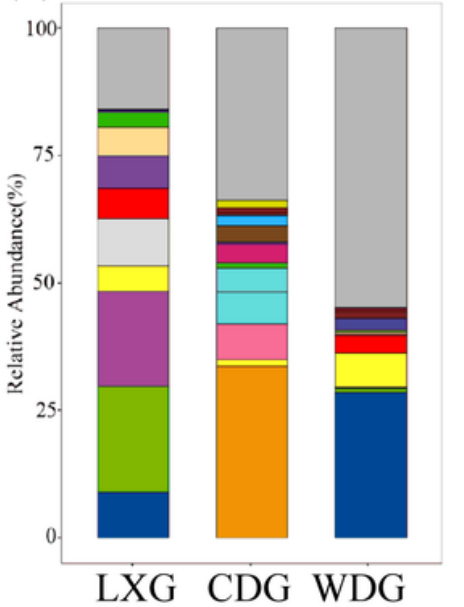

LXG CDG WDG
Others

Collembolispora

Trichoderma

Ganoderma

Rhodotorula

Humicola

Exophiala

Phoma

Rhodosporidis

Inocybe

Hygrocybe

Alternaria

Cladosporiun

Tomentella

Hygrophorus

Mortierella

Sebacina

Cortinarius
Fusarium

Russula
(B)

k_Fungi_g_Fusarium

$0.40-$

芦 0.30

龸 0.20

0.10

0 -

$\overline{\mathrm{LXG}} \quad \overline{\mathrm{CDG}} \quad \overline{\mathrm{WDG}}$
(C)

0.75 .

k_Fungi_g_Alternaria

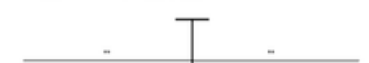

0 .

LXG

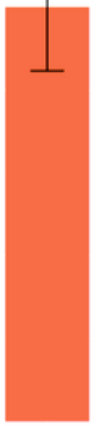

CDG

WDG

\section{Figure 4}

The genus level of fungal community composition. The relative abundance of Fusarium (B) and Alternaria $(A)$ in three types of ginseng rhizosphere, data were means \pm standard error. The relative abundances in the top 20 were chosen to exhibit. Others represented of low relative abundance that ranks lower than top 20. *Significant at the 0.05 probability level. ${ }^{*}$ Significant at the 0.01 probability level. 


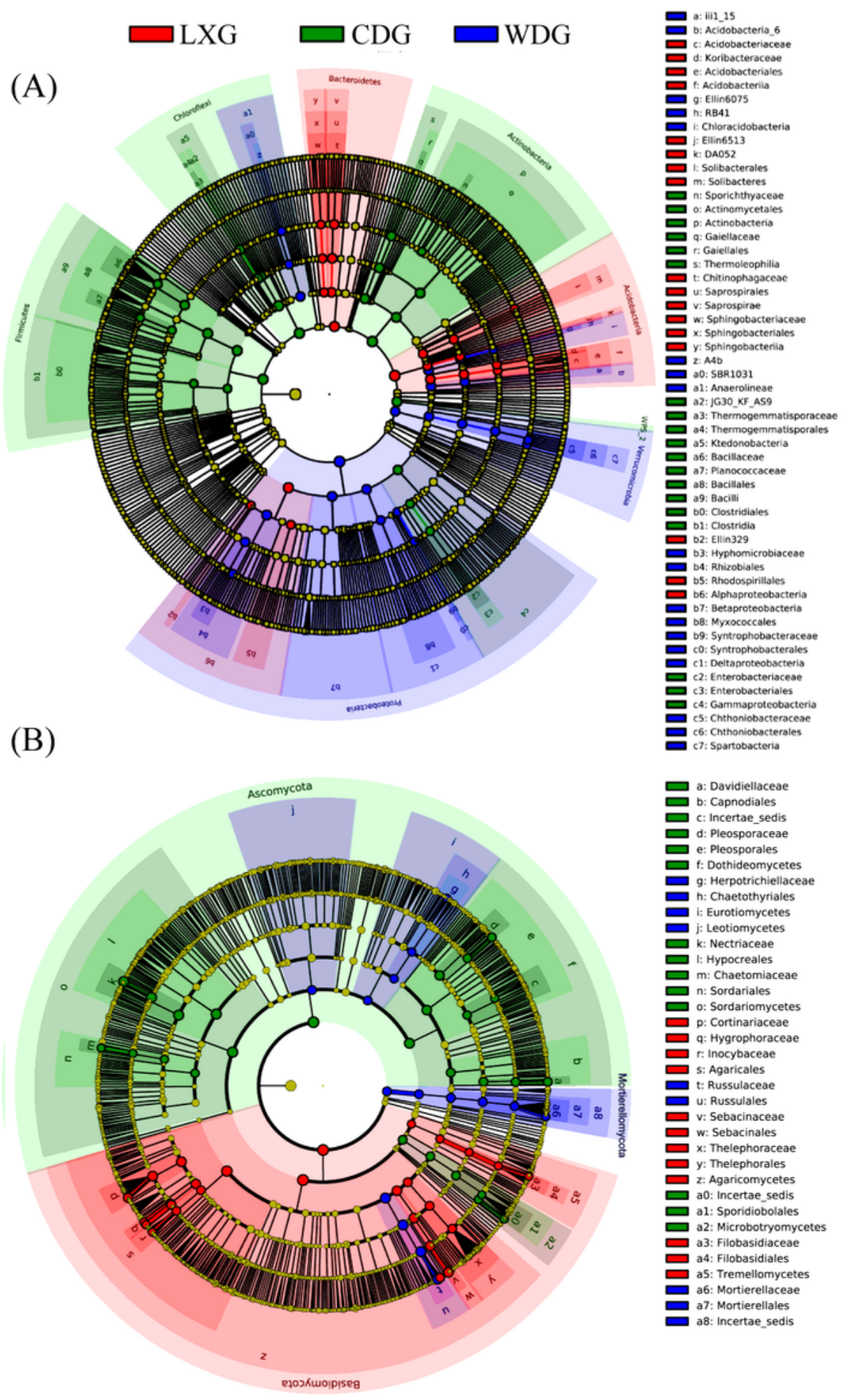

Figure 5

LEfSe analysis showing the different taxa among three growing status of ginseng rhizosphere in bacteria (A) and fungi (B). The diameter of each circle is proportional to the relative abundance of the taxon. The inner to outer circle corresponds to the level of the phylum to the genus.

\section{Supplementary Files}


This is a list of supplementary files associated with this preprint. Click to download.

- additionaltablefigure.docx 